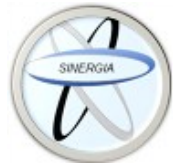

\title{
Carcinoma epidermoide cutáneo, enfermedad de alta relevancia mundial
}

\author{
Cutaneous squamous cell carcinoma, a disease of high worldwide relevance
}

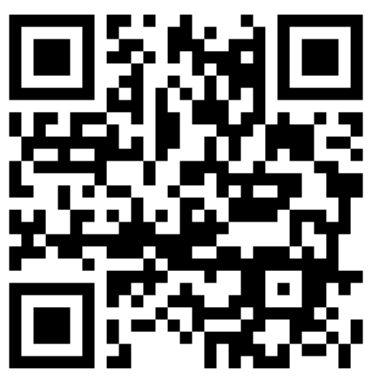

Recibido 08/09/2021
${ }^{1}$ Dr. José Roberto Hernández Chacón Hospital Nacional de Geriatría y Gerontología, San José, Costa Rica

(iD) https://orcid.org/0000-0002-1174-4887

${ }^{2}$ Dra. Sheirys Arleth Torres Morales Centro de Desarrollo Estratégico e Información en Salud y Seguridad Social (CENDEISSS), San José, Costa Rica (iD) https://orcid.org/0000-0003-0134-8175

${ }^{3}$ Dra. Evelyn María Hernández Chacón Centro de Desarrollo Estratégico e Información en Salud y Seguridad Social (CENDEISSS), San José, Costa Rica

(iD) https://orcid.org/0000-0001-5592-5703

Corregido 20/09/2021

Aceptado 20/10/2021

\section{RESUMEN}

El carcinoma epidermoide cutáneo (CEC), antiguamente llamado carcinoma espinocelular o escamoso se define como el tumor primario de la piel que expresa una diferenciación descamativa predominante. Suele aparecer a partir de lesiones precursoras, como la queratosis actínica o la enfermedad de Bowen (carcinoma in situ), aunque también puede aparecer en pieles irradiadas o sobre lesiones inflamatorias crónicas o de novo, tales como en cicatrices de quemaduras, úlceras en miembros inferiores y en casos de micosis profundas; así como en zonas de alto y medio riesgo de la cabeza y cuello. Tiene una incidencia hasta tres veces mayor en hombres que en mujeres, y se presenta principalmente entre las edades de 64 a 76 años. Es la segunda causa de muerte por cáncer cutáneo después del melanoma. Forma parte de los cánceres cutáneos no melanocíticos y se considera uno de los cánceres más frecuentes en la población de piel blanca a nivel mundial, por lo que el tener tipo de piel I o II según la clasificación Fitzpatrick y el tener labores al aire libre tales como agricultura y construcción, aumentan el riesgo de padecer la enfermedad. El diagnóstico definitivo del CEC 
se establece mediante biopsia de la lesión y examen con histopatología. La cirugía representa el tratamiento de elección y la técnica de Mohs se considera la técnica de elección.

PALABRAS CLAVE: carcinoma epidermoide de cabeza y cuello; carcinoma epidermoide oral; carcinoma epidermoide; carcinoma.

\section{ABSTRACT}

Cutaneous squamous cell carcinoma (SCC), formerly called squamous cell carcinoma, is defined as the primary skin tumor that expresses a predominant desquamative differentiation. It usually appears from precursor lesions, such as actinic keratosis or Bowen's disease (carcinoma in situ), although it can also appear on irradiated skin or on chronic or de novo inflammatory lesions, such as burn scars, ulcers on the lower limbs and in cases of deep mycosis; as well as in high and medium risk areas of the head and neck. It has an incidence up to three times more in men than in women, and it occurs mostly between the ages of 64 to 76 years. It's the second leading cause of death from skin cancer after melanoma. It's part of the non-melanocytic skin cancers and is considered one of the most frequent cancers in the white-skinned population, skin types I and II according to the Fitzpatrick classification and having work outdoors such as agriculture and construction, have a higher risk of suffering the disease. The definitive diagnosis of SCC is established by biopsy of the lesion and examination with histopathology. Surgery represents the treatment of choice and the Mohs technique is considered the technique of choice.

KEYWORDS: squamous cell carcinoma of head and neck; oral squamous cell carcinoma; squamous cell carcinoma; carcinoma.

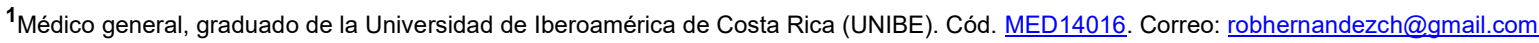

${ }^{2}$ Médica general, graduada de la Universidad de Iberoamérica de Costa Rica (UNIBE). Cód. MED14370. Correo: sheirystorres@gmail.com

${ }^{3}$ Médica general, graduada de la Universidad de Iberoamérica de Costa Rica (UNIBE). Cód. MED16718. Correo: evelmhch@gmail.com

\section{INTRODUCCIÓN}

El carcinoma epidermoide cutáneo (CEC), antiguamente llamado carcinoma espinocelular o escamoso, es un cáncer cutáneo con una alta repercusión en la salud pública. Se define como el tumor primario de la piel que expresa una diferenciación descamativa predominante, a diferencia de otros carcinomas cutáneos (1).

EI CEC suele aparecer a partir de lesiones precursoras, como la queratosis actínica o la enfermedad de Bowen (carcinoma in situ), aunque también puede aparecer en pieles irradiadas o sobre lesiones inflamatorias crónicas o de novo (1). El CEC es la segunda causa de muerte por cáncer cutáneo después del melanoma, provocando la mayoría de las muertes por cáncer de piel en adultos mayores de 85 años, e incluso se compara con la mortalidad provocada por otros carcinomas tales como renales, orofaríngeos o melanomas (2).

El número de casos ha incrementado entre el 50 y el $300 \%$ en las últimas 3 décadas y se espera que para el año 2030 en los países europeos se duplique (2). El CEC se origina a partir de queratinocitos epidérmicos 
y estructuras anexiales tales como glándulas ecrinas o unidades pilosebáceas. Además, es el cáncer cutáneo más frecuente que se forma dentro de una cicatriz ya existente (3). Al tratarse de una patología con alta relevancia mundial y al ser la segunda causa de muerte por cáncer cutáneo, se realiza una revisión bibliográfica que permita valorar los artículos y estudios más recientes sobre la enfermedad, con el objetivo de actualizar y refrescar conocimientos a los profesionales de la salud sobre el CEC.

\section{MÉTODO}

Se realiza una revisión bibliográfica de artículos y de estudios científicos relevantes, que den validez a lo planteado en el objetivo de esta investigación. Se buscaron estudios en los idiomas inglés y español, y publicados entre los años 2016 y 2021. Se utilizaron buscadores tales como Cochrane Database, Scientific Electronic Library Online (SciELO) y Medical Literature Analysis and Retrieval System Online (MEDLINE / PUBMED). Los descriptores empleados fueron "carcinoma", "carcinoma epidermoide cutáneo", "carcinoma epidermoide oral", "enfermedad bowen" y "cáncer no melanocitico" Para la selección de las publicaciones se analizó el texto completo y se identificaron los más relevantes para desarrollar el artículo. Al final del proceso de selección se escogieron 15 artículos.

\section{EPIDEMIOLOGÍA}

El CEC forma parte de los cánceres cutáneos no melanocíticos y se considera uno de los cánceres más frecuentes en la población de piel blanca a nivel mundial $(1,4-6)$. Tiene una incidencia hasta tres veces más en hombres que en mujeres, y se presenta con más frecuencia en personas con edades entre 64 y 76 años. Se han diagnosticado casos entre jóvenes de 20 a 30 años en algunas regiones de Australia, Nueva Zelanda y en los estados de California del Sur y Florida de Estados Unidos de América. La incidencia aumenta progresivamente desde los 30 años, esto se puede deber al envejecimiento de la población y a los hábitos de exposición solar $(1,3)$.

La incidencia de padecer la enfermedad va del 2,4 al $14 \%$ y puede aumentar hasta un $24 \%$ en los CEC recurrentes. Por otro lado, si afecta mucosas de la cabeza y cuello puede alcanzar hasta un $29 \%$. En cuanto al tamaño de la lesión, suele estar presente en el $64 \%$ de los tumores mayores de $2.5 \mathrm{~cm}$ y en el $11 \%$ de los tumores menores de $2.5 \mathrm{~cm}$ (7).

EI CEC tiene un riesgo de recidiva local del $8 \%$ y de metástasis ganglionar de entre un 2 y un $5 \%$. En el $80 \%$ de los casos, las metástasis afectan a los ganglios de la zona de drenaje, pero también puede metastatizar a órganos tales como el pulmón, hígado, cerebro, hueso, entre otros (1). El CEC metastásico ganglionar tiene una incidencia de un $2,4 \%$ en hombres y de un $1,1 \%$ en mujeres (8).

La supervivencia del CEC a 10 años posterior al tratamiento quirúrgico es de más del $90 \%$, la frecuencia de metástasis ganglionares está alrededor del $4 \%$ y la mortalidad es de un $2 \%$ aproximadamente $(1,2)$.

\section{FACTORES PRONÓSTICOS}

Entre los factores de riesgo se encuentran la exposición a la radiación ionizante, carcinógenos ambientales y lesiones precancerosas tales como las queratosis actínicas y úlceras crónicas. El factor más frecuente para enfermar por CEC es la 
exposición aguda o crónica a la radiación ultravioleta (UV) con una longitud de onda en el rango de los 290 a 320 nm (5). Éste afecta principalmente a personas menores de 20 años, y tiende a ser acumulativo, lo que incrementa la carcinogénesis en la edad adulta (4). Los tipos de piel I y II según la clasificación Fitzpatrick, el tener labores al aire libre tales como agricultura $y$ construcción, aumentan el riesgo de padecer la enfermedad $(3,9)$.

Se ha determinado que existe relación entre la enfermedad del CEC y el virus del papiloma humano (VPH), principalmente con los serotipos relacionados a lesiones malignas, como el tipo 16, 18, 31, entre otros $(5,10)$. Entre los factores predisponentes para un CEC en región anal se encuentran enfermedades de transmisión sexual, tales como clamidia, VPH, virus de inmunodeficiencia humana (VIH), condilomas acuminados, entre otros. En mujeres puede existir mayor riesgo en aquellas con antecedente de cáncer cervicouterino (11).

Entre los factores que aumentan el riesgo de padecer un carcinoma epidermoide verrugoso, se encuentra el tener contacto agentes químicos y físicos, como radiación ultravioleta, alcohol, tabaco, arsénico, radiaciones ionizantes, fototerapia; además lesiones tales como lo son las como úlceras, quemaduras, cicatrices, lupus eritematoso discoide y liquen plano (10). En usuarios tabaquistas y que consumen licor, se ha visto un aumento en el riesgo de padecer carcinoma epidermoide en cavidad oral (12). Los factores pronósticos se pueden clasificar en varios grupos, inicialmente se encuentran los factores relacionados con el tumor, por ejemplo, el tamaño horizontal del tumor primario, ya que si es superior a $2 \mathrm{~cm}$ de diámetro horizontal se asocia a un riesgo 3 veces mayor de recidiva local y 6 veces más alto de metástasis. También la localización de la lesión, ya que si se encuentra en el pabellón auricular y el labio inferior se duplica el riesgo de metástasis (2).

Otros se han relacionado con un mayor tamaño tumoral y mayor riesgo de infiltración perineural y linfovascular, además tiende a ser más agresivo que el tumor primario. La asociación de síntomas neurológicos es un marcador clínico de mal pronóstico y suele estar relacionado a la presencia de invasión perineural $(2,13)$. La invasión de nervios más gruesos o a distancia del foco principal del tumor independientemente de su diámetro, implica un peor pronóstico $(2,7)$.

Luego se encuentran los factores relacionados con el paciente, en donde se encuentra principalmente la inmunosupresión, ya sea por ser tratado con trasplante de órganos 0 padecer de enfermedades autoinmunes (2). En una persona inmunosupresa, el riesgo de presentar un CEC se eleva a 65 veces más (6). Por otro lado, existe cierta susceptibilidad genética para padecer la enfermedad en paciente con algunas comorbiliades de fondo, tales como xeroderma pigmentoso, síndrome de Gorlin Goltz, albinismo, epidermólisis bullosa congénita, síndrome Ferguson-Smith y epidermodisplasia verruciforme (5). La queratosis actínica es la lesión premaligna más frecuente, se estima que una de cada mil lesiones progresa a CEC, seguido de la queilitis actínica (3). Los factores pronósticos histológicos son los más importantes para el desarrollo de metástasis. Se ha visto que la probabilidad de metástasis es casi nula si el espesor del CEC es menor de $2 \mathrm{~mm}$, cerca del $6 \%$ en tumores de 2 a $6 \mathrm{~mm}$ y del $16 \%$ en tumores de más de $6 \mathrm{~mm}$ de espesor. A la vez, si se encuentra la invasión del tumor más allá de la grasa subcutánea, se ha 
relacionado con un riesgo 7 veces mayor de recidiva y 11 veces mayor de metástasis (2).

\section{HISTOLOGÍA}

Se pueden clasificar los CEC como bien diferenciados, moderadamente diferenciados y pobremente diferenciados, siendo los últimos los de peor pronóstico (13). Generalmente se reportan células pavimentosas de aspecto normal y atípico caracterizadas por aumento de mitosis, figuras mitóticas aberrantes, hipercromasia nuclear y pérdida de puentes intercelulares (5). Se caracteriza por presentar una proliferación de queratinocitos atípicos, que van desde la epidermis hasta la dermis, además presentan pleomorfismo celular y nuclear, nucléolos prominentes, células multinucleadas y figuras mitóticas atípicas (4).

En el caso del carcinoma verrugoso, a nivel histológico se observa una neoformación epitelial debido a la proliferación masiva de queratinocitos. En la capa córnea se presenta una intensa hiperqueratosis con focos de paraqueratosis y en la epidermis se proyecta hacia la superficie formando cordones de células tumorales que penetran la dermis y el tejido celular subcutáneo (13).

\section{CLÍNICA}

Según la clasificación de Peniche, el CEC puede presentarse como alguno de los siguientes 5 tipos: Ulceroso, nodular, nodular queratósico, vegetante y superficial (13).

La forma más frecuente es la ulcerosa, la cual se presenta como una lesión de base infiltrada, irregular y friable, crece de manera más rápida e infiltrativa, al compararse con el carcinoma basocelular (5).
La forma vegetante o verrugosa produce producir neoformaciones que pueden crecer a gran tamaño, ésta forma se ha encontrado en cicatrices de quemaduras, úlceras en miembros inferiores y en casos de micosis profundas (5). La mayoría de los casos de carcinoma epidermoide verrugoso se localizan en las plantas de los pies, principalmente la almohadilla plantar, seguida de los dedos y el talón (10)

Cuando el carcinoma cutáneo se desarrolla en el territorio de cabeza y cuello, suele ser más frecuentemente sobre la piel de las regiones geniana, malar, nasal, frontal, preauricular y cervical, conformando zonas de alto y medio riesgo (7). El CEC en cavidad oral se puede presentar a través de leucoplaquias, eritroleucoplaquias, eritroplaquia, leucoplaquia proliferativa verrucosa, fibrosis de la submucosa oral y liquen plano oral (12). El reconocer las lesiones potencialmente malignas en cavidad oral es relativamente sencillo en la exploración de la cavidad oral, pero el saber identificar y tratar lesiones de alto grado o pacientes de alto riesgo es uno de los principales retos (14).

La enfermedad de Bowen es sinónimo de carcinoma epidermoide in situ cutáneo, puede afectar la piel o las mucosas y tiene más incidencia después de los 70 años de edad. Suele presentarse como una placa eritematosa, bien delimitada y escamosa, generalmente es pigmentada, mal definida y plana. Es una lesión única, asintomática y fija. En su forma mucosa, se presenta como una placa única eritematosa lisa, levemente erosiva $y$ en ocasiones con bordes irregulares $(1,3)$. A nivel oftalmológico, en la conjuntiva se muestra con irritación de la misma, generalmente desarrollado posterior al uso crónico de lentes de contacto. Los pacientes detectan una masa conjuntival de gran tamaño en el área afectada. Si la lesión 
se presenta en el párpado, pueden asociarse síntomas oculares como disminución de la agudeza visual, diplopía, proptosis e irritación ocular (3). La eritroplasia de Queyrat (CEC in situ del pene) se describe como una lesión roja aterciopelada. En el caso que se relacione con el VPH se puede manifestar como un crecimiento verrugoso en el pene, la vulva, región perianal o periungueal (3). La primoinfección del carcinoma epidermoide de canal anal inicia con el VPH, entre los individuos con estilos de vida sexual precoz, desprotegida y/o promiscua. Inicia con un pólipo, el cual se transforma en cáncer posteriormente. Esta enfermedad es más común en mujeres que en hombres (11).

\section{DIAGNÓSTICO Y DIAGNÓSTICOS DIFERENCIALES}

El diagnóstico definitivo del CEC se establece mediante biopsia de la lesión y examen con histopatología (3).
En cuanto a los diagnósticos diferenciales de un CEC invasivo se incluyen todas aquellas afecciones dermatológicas tipo nódulos, placas y úlceras persistentes, principalmente en personas que tienen fotodaño, quemaduras antiguas, cicatrices o han recibido radiación. Entre estos, se encuentra la enfermedad de Bowen, el lupus eritematoso cutáneo crónico, el carcinoma basocelular superficial, la queratosis seborreica, tumor anexial y la verruga plana. El principal diagnóstico diferencial clínico histológico del CEC es el queratoacantoma $(1,3)$.

En una sospecha clínica de enfermedad de Bowen se debe realizar un diagnóstico diferencial con la mayoría de las dermatosis que pueden presentar placas eritematosas bien delimitadas, como el melanoma amelanocítico, la psoriasis, la enfermedad de Paget, condiloma acuminado, entre otros (3).

Figura 1. Imágenes de lesiones cutáneas de carcinoma de células escamosas
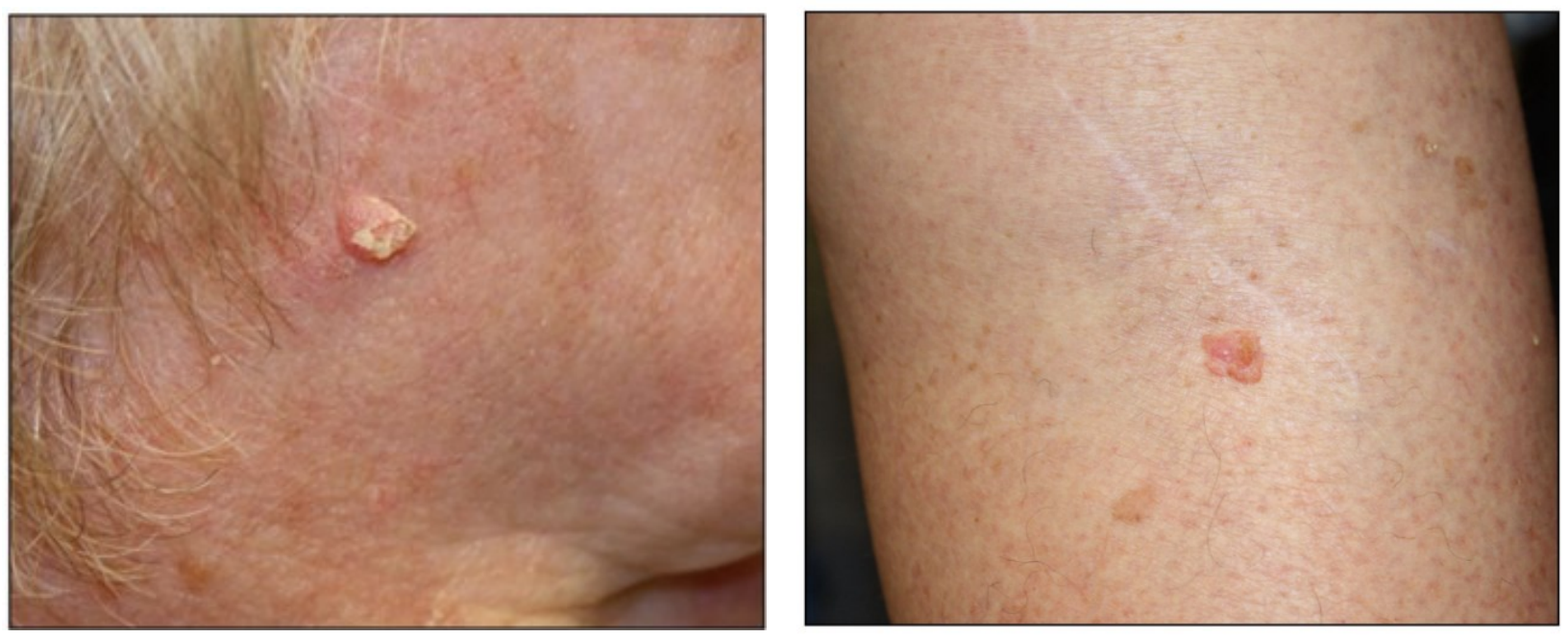

Imagen A. Lesión de cáncer de piel en la cara que se observa levantada y costrosa.

Imagen B. Lesión de cáncer de piel en la pierna que se ve rosa y levantada

Fuente. Cancer.gov. [cited 2021 Sep 1]. Available from: https://www.cancer.gov/espanol/tipos/piel/paciente/tratamiento-piel-pdq 


\section{TRATAMIENTO}

La cirugía representa el tratamiento de elección y la técnica de Mohs se considera la técnica de elección, ya que logra alcanzar tasas de recurrencia inferiores, y a la vez una mayor sensibilidad para la detección de una invasión perineuronal, ya que permite examinar los márgenes laterales y profundos en su totalidad, proporcionando la mejor semejanza de extirpación tumoral completa $(1,6,7)$.

Para los tumores de bajo grado se recomienda dar un margen de seguridad de entre 4 y $6 \mathrm{~mm}$ y hacer cierre primero o reconstruir con colgajo o injerto; para los tumores de alto grado, la técnica de elección es la de Mohs. Si esta técnica no se puede utilizar, se realiza la resección con un margen de un centímetro (6). Los estudios indican que las tasas de recurrencia local son del $47 \%$ para la cirugía convencional y del $0 \%$ para la cirugía de Mohs (7).

La radioterapia es útil en casos selectos, principalmente cuando el paciente rechaza la cirugía, en casos de tumores irresecables o en pacientes inoperables. Si las pruebas de imagen demuestran invasión perineural intracraneal o diseminación perineural, la lesión se puede considerar irresecable (7).

La radioterapia tiene tres funciones principales, primeramente, como tratamiento de primera línea con intención curativa, luego en tratamiento complementario a la cirugía y por último como tratamiento paliativo (15).

La radioterapia para el tratamiento de cáncer de piel tiene dos modalidades posibles, la primera es la radioterapia externa, que se constituye por 3 tipos: la macroterapia superficial Dermopan (fotones de ortovoltaje), electrones de acelerador lineal o fotones de alta energía. Es un tratamiento costoso, que requiere una infraestructura concreta, lo cual puede constituir otra limitación a su uso (15).

La radiación ionizante produce la muerte de las células tumorales más indiferenciadas y con mayor actividad mitótica. Este procedimiento no provoca la muerte de la célula, al contrario, afecta su capacidad mitótica luego de 2 o 3 ciclos celulares (15). Los tratamientos sistémicos que incrementan la radiosensibilidad pueden mejorar el control de la enfermedad frente a la radioterapia sola, en tumores localmente avanzados o regionalmente metastásicos (15).

\section{CONCLUSIONES}

EI CEC es un cáncer cutáneo que presenta una alta repercusión en la salud pública a nivel mundial, ya que es la segunda causa de muerte por cáncer cutáneo, seguido del melanoma y afecta mayormente a adultos mayores de 85 años. Se espera, en menos de diez años, la incidencia por dicha enfermedad se duplique.

Es una enfermedad altamente prevenible, ya que el mayor factor de riesgo es la exposición a la radiación ultravioleta. Se debe educar a la población sobre los métodos de prevención del CEC, mediante medios de protección solar, tales como filtro solar, ropa encubridora, paraguas, sombreros, entre otros. Es importante educar a los pacientes sobre la importancia de evitar la exposición al sol, y así reducir el riesgo de presentar la enfermedad.

Entre los principales lugares en donde se puede producir el CEC, se encuentran zonas de cicatrices de quemaduras, úlceras en miembros inferiores y en casos de micosis profundas, generalmente en las plantas de los pies; en el territorio de cabeza y cuello, más frecuentemente en zonas de moderado y alto riesgo. El diagnóstico definitivo se 
realiza mediante biopsia de la lesión y valoración histopatológica y el tratamiento de elección es la cirugía, la técnica de Mohs se considera la técnica de elección ya que logra obtener tasas de recurrencias menores que las demás. La radioterapia se utiliza en pacientes a los que no se les pueda realizar cirugía, ya sea por rechazo del paciente o por ser tumores irresecables; funciona tanto como tratamiento de primera línea en los antes mencionados, como en tratamiento complementario o paliativo.

\section{Los autores declaran no tener conflicto de interés.}

\section{REFERENCIAS}

1. Becquart $\mathrm{O}$, Guillot B. Carcinoma epidermoide cutáneo y sus precursores. EMC - Dermatología [Internet]. 2020;54(1):1-19. Disponible en: https://doi.org/10.1016/S1761-2896(20)43360-6

2. Cañueto J, Tejera-Vaquerizo $A$, Redondo $P$, Botella-Estrada R, Puig S, Sanmartin O. A review of terms used to define cutaneous squamous cell carcinoma with a poor prognosis. Actas Dermosifiliogr. 2020;111(4):281-90.

3. Kallini JR, Hamed N, Khachemoune A. Squamous cell carcinoma of the skin: Epidemiology, classification, management, and novel trends. Int J Dermatol. 2015;54(2):130-40.

4. Soria LA, García Massó D, Chacón Oduardo E. Presentación clínica atípica del carcinoma epidermoide cutáneo Atypical Clinical Presentation of Cutaneous Squamous Cell. Rev Cuba Med Miliar. 2016;45(2):215-20.

5. Rodríguez Beltrán S, Reynoso von Dratlen C. Cáncer epidermoide en piel cabelluda, presentación inusual. Reporte un caso. Rev la Fac Med la UNAM. 2017;60(2):17-21.

6. Domínguez-Cherit J, Rodríguez-Gutiérrez G, Narváez Rosales V, Toussaint Caire S, Fonte Avalos V. Characteristics and risk factors for recurrence of cutaneous squamous cell carcinoma with conventional surgery and surgery with delayed intraoperative margin assessment. Cir y Cir (English Ed [Internet]. 2017;85(6):499-503. Disponible en: http://dx.doi.org/10.1016/j.circir.2016.11.013

7. Pérez García MP, Mateu Puchades A, Sanmartín Jiménez $O$. Perineural Invasion in Cutaneous
Squamous Cell Carcinoma. Actas Dermosifiliogr [Internet]. 2019;110(6):426-33. Disponible en: https://doi.org/10.1016/j.ad.2018.10.006

8. Villegas-Romero I, Jiménez-Gallo D, GutiérrezBayard L, Linares-Barrios M. Advanced Cutaneous Squamous Cell Carcinoma Treated with Pembrolizumab. Actas Dermosifiliogr. 2021;112(7):672-5.

9. Sandoval Pérez JH, Insfrán WN, Aldana Ubilus CA. Epidermodisplasia verruciforme y carcinoma epidermoide - diagnóstico y tratamiento. Fac Cienc Méd [Internet]. 2020;53(3):2020. Disponible en:

http://dx.doi.org/10.18004/anales/2020.053.02.15 7

10. Aguinaga-inurriaga $\mathrm{AE} \mathrm{De}$, Xospa-ríos VI, Messina-robles $M$, Sierra-díaz CG, Aguinagainurriaga AE De, Xospa-ríos VI, et al. Carcinoma epidermoide verrugoso asociado con infección por virus del papiloma humano. Dermatol Rev Mex. 2018;62(2):137-43.

11. Santos Toledo Y, Estepa Pérez JL, Feliú Rosa JA, Santana Pedraza T, Díaz Conde DA. Caracterización de pacientes diagnosticados de carcinoma epidermoide de canal anal. Cienfuegos, 2017-2019. Medisur. 2021;19(1):2017-9.

12. Farooq I, Bugshan A. Oral squamous cell carcinoma: Metastasis, potentially associated malignant disorders, etiology and recent advancements in diagnosis. F1000Research. 2020;9:1-10.

13. Morales López O, Garduño Mejía JE. Carcinoma epidermoide cutáneo recidivante en región frontal: revisión de la literatura y reporte de caso clínico. Rev la Asoc Dent Mex. 2017;74(5):239-44.

14. Thomson PJ. Perspectives on oral squamous cell carcinoma prevention-proliferation, position, progression and prediction. J Oral Pathol Med. 2018;47(9):803-7.

15. Cañueto J, Jaka A, Toll A. The Value of Adjuvant Radiotherapy in Cutaneous Squamous Cell Carcinoma: A Review. Actas Dermosifiliogr. 2018;109(6):476-84. 